\title{
Viscous Self Interacting Dark Matter Cosmology For Small Redshift
}

\author{
Abhishek Atreya, ${ }^{1, *}$ Jitesh R. Bhatt, ${ }^{2, \dagger}$ and Arvind Kumar Mishra ${ }^{2,3, \ddagger}$ \\ ${ }^{1}$ Center For Astroparticle Physics and Space Sciences, \\ Bose Institute, Kolkata, 700009, India \\ ${ }^{2}$ Theoretical Physics Division, Physical Research Laboratory, \\ Navrangpura, Ahmedabad, 380009, India \\ ${ }^{3}$ Indian Institute of Technology, Gandhinagar, 382424, India
}

\begin{abstract}
The viscosity of dark matter in cosmological models may cause an accelerated expansion and when this effect is sufficiently large, it can explain the dark energy. In this work, attributing the origin of viscosity to self-interaction of dark matter, we study the viscous cosmology at small redshift $(0 \leq z \leq 2.5)$. Assuming the cluster scale to be virialized and by modeling a power law behavior of velocity gradients, we calculate the Hubble expansion rate, $H(z)$ and the deceleration parameter, $q(z)$. We then perform a $\chi^{2}$ analysis to estimate the best fit model parameters. By using the best fit values, we explain the cosmic chronometer and type Ia supernova data. We conclude that if the dissipative effects become prominent only at the late time of cosmic evolution and are smaller at higher redshift, we can explain the observational data without requiring any dark energy component. Our analysis is independent of any specific model of self interacting dark matter.
\end{abstract}

PACS numbers:

*Electronic address: atreya.abhi@gmail.com

$\dagger$ Electronic address: jeet@prl.res.in

${ }^{\ddagger}$ Electronic address: arvind@prl.res.in 


\section{INTRODUCTION}

The observations of large redshift supernovae provide a compelling evidence that the Universe has gone in an accelerating phase lately [1,2]. To explain the observations, a hypothetical new form of energy, called the dark energy (DE), is required which contributes $\sim 70 \%$ of the total energy budget of the Universe. Rest $\sim 30 \%$ of the energy density comes from the baryonic matter and an unknown form of matter, called dark matter (DM). Several other independent observations such as Baryon Acoustic Oscillations (BAO) [3], Cosmic Microwave Background Radiation (CMBR) [4], Large Scale Structures (LSS) [5] etc. provide concrete support to the above picture.

Many theoretical attempts have been made to explain dark energy either by modifying the gravity sector or the energy momentum content of the Einstein's field equation, see review $[6,7]$. The cosmological constant $\Lambda$, is the most successful model to explain dark energy but there is no theory to explain its physical origin and its present value, see Ref. [8].

It is a possibility that the dark energy is not a separate entity but a manifestation of some intrinsic properties of dark matter. It has been argued that if cosmic fluid is not perfect but viscous, then its effective pressure, $P+\Pi_{B}$ may turn negative and change the solution of Einstein's equation, thereby affecting the cosmic evolution of the Universe, leading to an inflation like behaviour [9-12]. Similar arguments have been made in the context of currently observed cosmic acceleration [13-16]. It have been also shown that the bulk viscosity can unify the dark sectors. i.e. dark matter and dark energy. The source of bulk viscosity has been attributed to neutrinos [17], an exotic scalar field [18] or the decay of cold dark matter into relativistic particles [19]. However these model are highly constrained by CMB and LSS observations, as large bulk viscosity reduces the gravitational potential, which in turn affect the structure formation [20-23]. For recent discussions on the role of cosmic viscosity, we refer to [24-29].

However it has been argued in Ref. [30] that at late times, both bulk and shear viscosity of cosmic fluid might play an important role in cosmic expansion and contribute to the observed accelerated expansion of the Universe without any dark energy. In our previous work [31], we argued that dark matter self-interactions can produce sufficient dissipation that can explain the present cosmic acceleration. The motivation for SIDM comes from 
the small scale astrophysical observations which demand some self-interactions between the dark matter particles $[32,33]$. Due to self-interactions it is natural to expect some non-zero viscosity in the dark matter sector. Also SIDM does not conflict with the LSS observation, as it behaves like an interacting matter at small scales and a non-interacting matter at large scales.

We derive the expressions for viscous coefficients (shear and bulk viscosity) of SIDM using the kinetic theory formalism and estimate the bulk and shear viscosity due to SIDM. To estimate the viscosity, we use the value of $\langle\sigma v\rangle / m$ as obtained in Ref. [34] by utilizing the astrophysical data from dwarf galaxies, Low Surface Brightness (LSB) galaxies and clusters. Further we estimate the mean free path of SIDM $\lambda_{\text {SIDM }} \sim 1 \mathrm{Mpc}$, which is order of cluster scale and argue that the cluster scale is smallest scale where the hydrodynamics is valid. We concluded that the viscous effects of the SIDM are sufficient enough to account for the present observed accelerated expansion of the Universe without any extra dark energy component [31].

In this work we extend our previous study to look at the late time dynamical evolution of universe within the framework of viscous SIDM cosmology without any dark energy component [31]. An important assumption in our analysis is that in recent times the clusters size dark matter halos have virialized and thus these are the relevant scales for estimating viscosity. At larger scales the velocity perturbations evolve and the viscous effects thus manifest themselves at those scales by contributing to energy dissipation. To calculate the dissipation term, we consider a few simplified assumptions. Assuming a power law parameterization of average velocity gradient on the redshift and with our estimates of viscosity of SIDM from [31], we set-up the equations for the Hubble parameter $H(z)$ and deceleration parameter $q(z)$.

To extract the values of power law exponent and length scale, we use the $\chi^{2}$ analysis with the cosmic chronometer data and find that the best fit values also explain the supernova data. The best fit values extracted from the fit dictate that dissipation was smaller at earlier times which is consistent with the expectation that the gradients become prominent only at late times thus affecting the cosmic evolution. We also extract the epoch of decelerationacceleration transition and find that the value of deceleration parameter approaches $q \sim 0.5$, for the best fit values, in the matter dominated era as expected.

The arrangement of our work is as follows: In section II, using the kinetic theory formalism 
we calculate the viscosity of SIDM and discuss its effect on the cosmic evolution. In section III, following some simplifying assumptions, we approximate the form of dissipation term $D$. By using the from of $D$, we then set up the coupled differential equation for the Hubble rate and deceleration parameter. In section IV we perform the $\chi^{2}$ analysis with the cosmic chronometer data and estimate the best fit model parameter of viscous SIDM model. In section V, we present our results. The best fit values successfully explain supernova data. We also discuss the evolution of deceleration parameter and dissipation due to viscous effects. In the last section VI, we summarize and conclude our work.

Throughout the paper, we adopt the convention for denoting differentiation $\dot{A} \equiv \frac{d A}{d \tau}$ and $A^{\prime} \equiv \frac{d A}{d z}$.

\section{VISCOSITY OF SIDM AND COSMIC ACCELERATION}

In this section, we estimate the viscosity of SIDM due to self interactions, using kinetic theory [31]. We then use it to estimate the corrections to the Einstein equations.

\section{A. Viscosity of SIDM}

The starting point of kinetic theory is Boltzmann's equation

$$
\frac{\partial f_{p}}{\partial t}+v_{p}^{i} \frac{\partial f_{p}}{\partial x^{i}}=I\left\{f_{p}\right\}
$$

where $\mathbf{v}_{p}$ is single particle velocity, $f_{p}$ is the distribution function and $I\left\{f_{p}\right\}$ is collisional term. Within the "relaxation time formalism", we can approximate the change in the distribution function due to collisions as

$$
\delta f_{p}=-\tau\left(\frac{\partial f_{p}^{0}}{\partial t}+v_{p}^{i} \frac{\partial f_{p}^{0}}{\partial x^{i}}\right) .
$$

where $\tau$ is the relaxation time. It was argued in Ref. [31] that this is a valid approximation for dark matter halos. Since $\delta f_{p}$ is the deviation from the equilibrium distribution, the dissipative part of total energy momentum tensor, $T^{\mu \nu}=T_{\text {Ideal }}^{\mu \nu}+T_{\text {Diss }}^{\mu \nu}$, is given by

$$
T_{\text {Diss }}^{i j}=\int \frac{d^{3} p}{(2 \pi)^{3}} v^{i} p^{j} \delta f_{p}
$$

Comparing with the dissipative component of $T^{\mu \nu}$ in viscous hydrodynamics one finds [35, 36]

$$
\eta=\frac{1}{15 T} \int \frac{d^{3} p}{(2 \pi)^{3}} \tau \frac{p^{4}}{E_{p}^{2}} \frac{\partial f_{p}^{0}}{\partial E_{p}},
$$




$$
\text { and } \quad \zeta=\frac{1}{T} \int \frac{d^{3} p}{(2 \pi)^{3}} \tau\left[E_{p} C_{n}^{2}-\frac{p^{2}}{3 E_{p}}\right]^{2} f_{p}^{0} .
$$

where $C_{n}=\left.\frac{\partial P}{\partial \epsilon}\right|_{n}$ is the speed of sound at constant number density.

The exact expression for $\tau$ will depend on the specific model of SIDM. To keep our analysis model independent, we approximate relaxation time $\tau$, by its thermal average

$$
\tilde{\tau}^{-1}=n\langle\sigma v\rangle
$$

where $n,\langle\sigma v\rangle$ are the average number density and velocity weighted cross-section average respectively. Using the non-relativistic Maxwell-Boltzmann distribution in fluid rest frame, with eq. (6), in the non-relativistic limit of eq. (4) and (5) we get [31]

$$
\begin{gathered}
\eta=\frac{1.18 m\langle v\rangle^{2}}{3\langle\sigma v\rangle} . \\
\zeta=\frac{5.9 m\langle v\rangle^{2}}{9\langle\sigma v\rangle} .
\end{gathered}
$$

We have used equipartition of energy to relate root mean square velocity with the temperature $T$ in deriving eq. (7) and (8). For more details on the calculations, see Ref. [31].

\section{B. Einstein's Equation With Viscosity}

In this section, we investigate the effects of viscosity of SIDM on the solution of Einstein's equation [30, 31]. We consider that our Universe consists of the viscous dark matter with no extra dark energy component.

The starting point is the total energy momentum tensor $T^{\mu \nu}$ of viscous fluid. In the Landau frame, one can write the energy momentum tensor for the viscous dark matter in the first order gradient expansion as

$$
T^{\mu \nu}=\epsilon u^{\mu} u^{\nu}+\left(P+\Pi_{B}\right) \Delta^{\mu \nu}+\Pi^{\mu \nu},
$$

where $\Delta^{\mu \nu}=u^{\mu} u^{\nu}+g^{\mu \nu}$ is the projection operator and $\Pi_{B}, \Pi^{\mu \nu}$ represent bulk stress and shear stress tensor respectively, defined as

$$
\begin{gathered}
\Pi_{B}=-\zeta \nabla_{\mu} u^{\mu} \\
\text { and } \Pi^{\mu \nu}=-\eta\left[\Delta^{\mu \alpha} \Delta^{\nu \beta}+\Delta^{\mu \beta} \Delta^{\nu \alpha}-\frac{2}{3} \Delta^{\mu \nu} \Delta^{\alpha \beta}\right] \nabla_{\alpha} u_{\beta}
\end{gathered}
$$


where $\eta$ and $\zeta$ represents shear and bulk viscosity of SIDM. The shear stress satisfies the conditions, $u_{\mu} \Pi^{\mu \nu}=0$ and $\Pi_{\mu}^{\mu}=0$.

The covariant energy momentum conservation is

$$
\nabla_{\mu} T^{\mu \nu}=0
$$

Considering the scalar perturbations in the metric and solving for average energy density under small fluid velocity approximation, i.e. $\bar{v}^{2} \ll 1$, we get the energy density evolution as $[30]$

$$
\frac{1}{a}\langle\dot{\epsilon}\rangle_{s}+3 H\left[\langle\epsilon\rangle_{s}+\langle P\rangle_{s}-3\langle\zeta\rangle_{s} H\right]=D
$$

where, $D=\frac{1}{a^{2}}\left\langle\eta\left[\partial_{i} v_{j} \partial_{i} v_{j}+\partial_{i} v_{j} \partial_{j} v_{i}-\frac{2}{3} \partial_{i} v_{i} \partial_{j} v_{j}\right]\right\rangle_{s}+\frac{1}{a^{2}}\left\langle\zeta[\vec{\nabla} \cdot \vec{v}]^{2}\right\rangle_{s}+\frac{1}{a}\langle\vec{v} \cdot \vec{\nabla}(P-6 \zeta H)\rangle_{s}$.

In the above expression $\langle A\rangle_{s}$ represent the spatial average of $A$. Here we see that the evolution of the average energy density $\left(\langle\epsilon\rangle_{s}\right)$ crucially depends on the dissipation term $D$. In addition, we need Einstein's equation to get the equation for the Hubble expansion rate. For this purpose we use the spatial average of the trace of Einstein equation $\left\langle G_{\mu}^{\mu}\right\rangle_{s}=-8 \pi G\left\langle T_{\mu}^{\mu}\right\rangle$. For equation of state (EoS) we define $\langle P\rangle_{s}+\left\langle\Pi_{B}\right\rangle_{s}=\hat{w}_{\text {eff }}\langle\epsilon\rangle_{s}$, and hence we get [30]

$$
\frac{\dot{H}}{a}+2 H^{2}=\frac{4 \pi G\langle\epsilon\rangle_{s}}{3}\left(1-3 \hat{w}_{\mathrm{eff}}\right) .
$$

Here we find that the dynamics of the energy density and Hubble rate depends on the $D$ and $\operatorname{EoS}\left(\hat{w}_{\text {eff }}\right)$.

\section{ESTIMATION OF DISSIPATION AND HUBBLE PARAMETER}

In this section, we expand upon the ideas discussed in section II to get an estimate of the dissipation $D$ and set up the set of equations we solve to get the evolution of universe.

\section{A. Estimating Dissipation $D$}

To get the Hubble expansion we need to estimate the extent of dissipation due to the viscosity of SIDM. To estimate $D$ we take following steps:

( $i$ ) Viscous coefficients $\eta, \zeta$ depend on the thermal averaged quantities and hence they depend on the scale over which the thermalization has happened. 
(ii) To estimate the length scale over which the average needs to be taken for viscosity calculation, we make the estimates for mean free path. In dilute gas approximation we have $\eta=\rho v \lambda / 3$. Using (7) we get $\lambda \sim 10^{10}(1 / \rho)(\mathrm{m} / \sigma) \mathrm{kpc}$, where $\sigma / m$ is in $\mathrm{cm}^{2} / \mathrm{g}$ and $\rho$ is in $\mathrm{M}_{\odot} \mathrm{kpc}^{-3}$ [31]. The $\sigma / m$ estimates for the galactic and cluster scales are $\sim 2 \mathrm{~cm}^{2} / \mathrm{g}$ and 0.1 $\mathrm{cm}^{2} / \mathrm{g}$ respectively [34]. The densities for the galactic scales are in the range $\sim 10^{6}-10^{8}$ $\mathrm{M}_{\odot} \mathrm{kpc}^{-3}[37,38]$. This gives mean free path $\sim 1 \mathrm{Mpc}$. This is much larger than galactic size $(\sim 10 \mathrm{kpc})$. For clusters, the densities are $\sim 10^{8} \mathrm{M}_{\odot} \mathrm{kpc}^{-3}[39,40]$ and we get $\lambda \sim 1$ Mpc [31]. This means that it is reasonable to assume that dark matter has undergone some interactions within a cluster size halo. Thus we assume the cluster size to be the smallest scales where averaging needs to done for estimating viscosity. Below this scale the hydrodynamic description doesn't hold.

(iii) We assume that dark matter has virialized on this length scale $(\sim 1 \mathrm{Mpc})$ much before our range of interest of redshift $(0 \leq z \leq 2.5)$ and hence we can assume $\eta$ and $\zeta$ to be constant during the evolution of the universe in this redshift range. This assumption breaks down as one goes to the epoch of structure formation but that is beyond the scope of the present work. To calculate the viscosity, we assume the typical cluster scale velocity $v \sim \frac{10^{-2}}{3}$ and for $\frac{\langle\sigma v\rangle}{m}$ we use the constraint obtained on the cluster scale as discussed in the Ref. [34]. We have incorporated the case where viscosity changes with the redshift in our ongoing work [41].

(iv) We assume that velocity derivatives are prominent and evolve at a scale $L$. Thus the scale $L$ should be larger than the scale at which we estimate viscosity. $L$, in principle, can be any scale between the disspative scale (where viscosity appear $\sim 1 \mathrm{Mpc}$ ) to the super cluster scale $(\sim 100 \mathrm{Mpc})$ where cosmic expansion becomes prominent.

$(v)$ We replace the peculiar velocity gradient $\partial v$ by its average spatial value, i.e. $\partial v \sim$ $\langle\partial v\rangle_{s}$. Since the velocity gradients evolve during the cosmic evolution, we make an ansatz for the space average peculiar velocity gradient over the smaller redshift $(0 \leq z \leq 2.5)$

$$
\langle\partial v\rangle_{s} \sim \frac{v_{0}}{L}(1+z)^{-n},
$$

where $n \geq 0$ is the free parameter. At present $\langle\partial v\rangle_{s}(z=0) \sim v_{0} / L$. The parameter $v_{0}$ is the value of velocity on the scale larger than the cluster scale, hence we can assume its value of supercluster scale velocity, i.e. $v_{0} \sim 6 v$. 
In the light of the above assumptions, we may approximate $D$, given by eq. (13b), as

$$
D=(1+z)^{2}\left(\frac{v_{0}}{L(1+z)^{n}}\right)^{2}\left(\frac{4}{3} \eta+2 \zeta\right) .
$$

From the eq. (16) we see that $D$ depends on the scale where derivatives are most prominent $(L)$, velocity at scale $L\left(v_{0}\right)$, viscous coefficients $(\eta, \zeta)$ and redshift $(z)$. It is clear from eq. (16) that in $D$, the contribution from the smaller scale (where inhomogeneities dominate) is large whereas contribution from the larger scale (where universe is more or less homogeneous) is small.

\section{B. Hubble Rate And Deceleration Parameter}

We now set up the equations for the evolution of Hubble expansion rate and deceleration parameter. In term of redshift $(z)$, the deceleration parameter $q$, is given by

$$
q(z)=-1+(1+z) \frac{H^{\prime}}{H}
$$

Using the dimensionless parameter $\bar{H}=H / H_{0}$, where $H_{0}$ is the value of Hubble parameter at $z=0$, the eq. (17) can be rewritten as

$$
\frac{d \bar{H}}{d z}=\frac{(q+1) \bar{H}}{(1+z)}
$$

The equation for deceleration parameter can be obtained by using the equations (13a) and

$$
-\frac{d q}{d \ln a}+2(q-1)\left(q-\frac{\left(1+3 \hat{w}_{\mathrm{eff}}\right)}{2}\right)=\frac{4 \pi G D\left(1-3 \hat{w}_{\mathrm{eff}}\right)}{3 H^{3}}
$$

It was argued in Ref. [31] that for the SIDM $\hat{w}_{\text {eff }} \sim 0$. Hence the evolution eq. for $q$, in term of the redshift is written as

$$
\frac{d q}{d z}+\frac{(q-1)(2 q-1)}{(1+z)}=\frac{4 \pi G D}{3(1+z) H^{3}}
$$

where $D$ is given by eq. (16). In terms of the dimensionless parameter $\bar{H}$, the above equation can be rewritten as

$$
\frac{d q}{d z}+\frac{(q-1)(2 q-1)}{(1+z)}=\beta\left(\frac{1+z}{\bar{H}^{3}}\right)
$$

where

$$
\beta=\frac{4 \pi G}{3 H_{0}^{3}}\left(\frac{4}{3} \eta+2 \zeta\right)\left(\frac{v_{0}}{L(1+z)^{n}}\right)^{2} .
$$




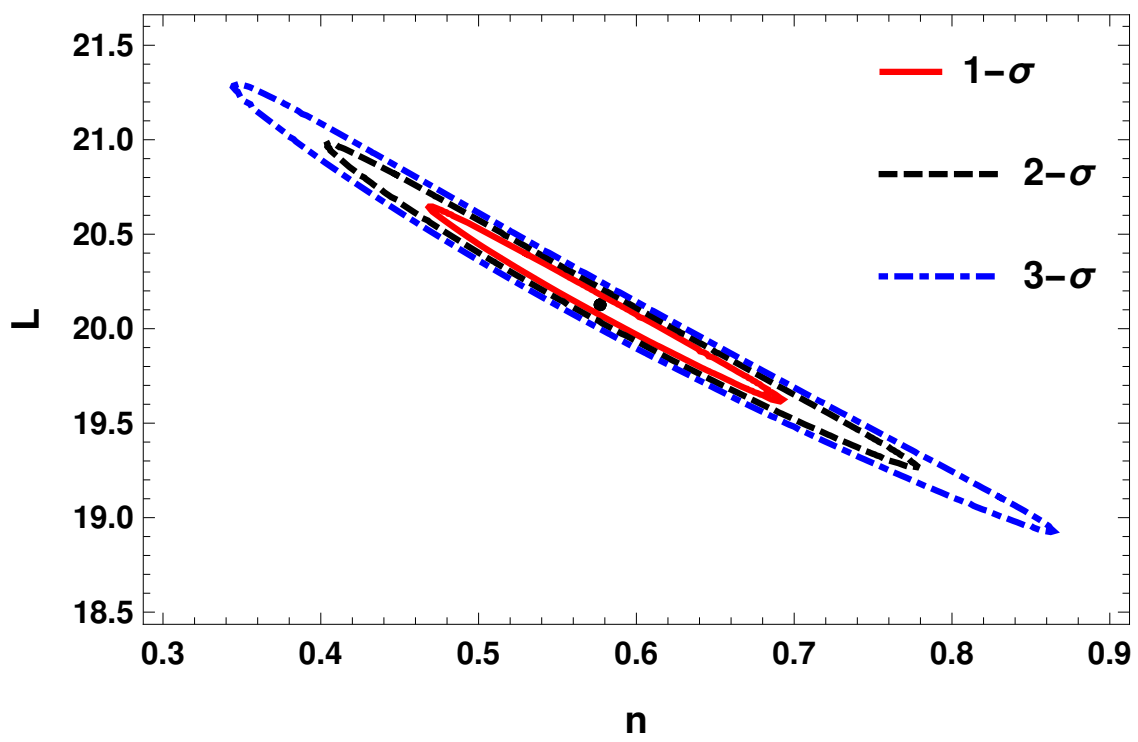

FIG. 1: The joint confidence region of model parameters $n$ and $L$ have been plotted. The region correspond to $68.3 \%, 95.4 \%$ and $99.73 \%$ confidence limits. The best fit value is shown as a point.

From eqn. (21) it is clear that the evolution of $q$ depends on $\beta$, the new dissipation parameter.

Eqns. (18) and (21) are coupled differential equation in $q(z)$ and $\bar{H}(z)$ that need to solve numerically. The initial condition for $\bar{H}(z)$ and $q(z)$ are given by its present value i.e $\bar{H}(z=0)=1$ and $q_{0}=-0.60$ (from CMB observations) [42].

\section{ESTIMATION OF MODEL PARAMETER USING COSMIC CHRONOME- TER DATA}

As we have seen in section IIIB, the solution for $\bar{H}(z)$ and $q(z)$, depends on two free model parameters $n$ and $L$. In this section, we will estimate the best fit value of model parameters $n$ and $L$ of viscous SIDM model using the $\chi^{2}$ minimization.

The theoretical model of the Hubble rate for viscous SIDM cosmology is given by the coupled differential eqns. (18) and (21). We find that Hubble rate depends on two free parameters i.e. $H(z, n, L)$. We use data for Hubble expansion rate from the cosmic chronometer data set given in Ref. [43] and reference therein.

The $\chi^{2}$ is defined as

$$
\chi^{2}(z, n, L)=\sum_{i=1}^{N}\left[\frac{H_{o b s}\left(z_{i}\right)-H_{t h}\left(z_{i}, n, L\right)}{\sigma_{i}}\right]^{2},
$$


TABLE I: The best fit model parameter

\begin{tabular}{||c|c|c|c|c||}
\hline Data set & $1-\sigma$ & $\chi_{\text {min }}^{2}$ & $\chi_{\text {d.o.f }}^{2}$ & Best fit model parameters \\
\hline \hline Cosmic chronometer & $0.5770_{-0.0679}^{+0.0766}$ & 22.02 & 0.61 & $n=0.5770$ \\
& $20.1265_{-0.3393}^{+0.0766}$ & & & $L=20.1265$ \\
\hline
\end{tabular}

where $N$ is the total number of cosmic chronometer data points and $\sigma_{i}^{2}$ is the variance in the $i$ th data points. Here $H_{o b s}\left(z_{i}\right)$ and $H_{t h}\left(z_{i}, n, L\right) \equiv H_{0} \bar{H}_{t h}\left(z_{i}, n, L\right)$ represents $i$ th observational Hubble parameter data and the theoretically predicted value for Hubble parameter respectively. The best fit value of the model parameters $n$ and $L$ have been estimated using the $\chi^{2}$ minimization.

The $\chi^{2}$ per degree of freedom, $\chi_{\text {d.o.f }}^{2}$, is given by $\frac{\chi_{\min }}{N-M}$, where $M$ is the number of parameters in the model. In our case $N=38$ and $M=2$. The best fit value of model parameters, $\chi_{\text {d.o.f }}^{2}$ and 1- $\sigma$ confidence region values are given in the table I. The contour plot of joint confidence region of model parameters $n$ and $L$ corresponding to $68.3 \%, 95.4 \%$ and $99.73 \%$ have also been plotted in the Fig. 1.

The best fit values are $n=0.5770$ and $L=20.1265 \mathrm{Mpc}$ for the power law exponent and the gradient length scale. We see that $L$ is approximately an order of magnitude larger than the cluster size scale $(\sim \mathrm{Mpc})$, which is the smallest scale for viscosity estimation.

\section{RESULTS}

In this section, we show the our results using the best fit model parameters and also compare with the constant dissipation $n=0$ prediction at the same length scale, i.e. $L=$ 20.1265 Mpc.

\section{A. Hubble expansion rate}

The plot for Hubble rate, obtained by numerically solving eqns. (18) and (21) and using the best fit values of parameters is given in Fig. 2. We also compare it with the case of constant dissipation and $\Lambda$ CDM model.

We see that constant dissipation case explain the data upto $z \leq 0.7$ and on larger redshift, Hubble start increasing drastically and fails to fit the data. On the other hand, the best 


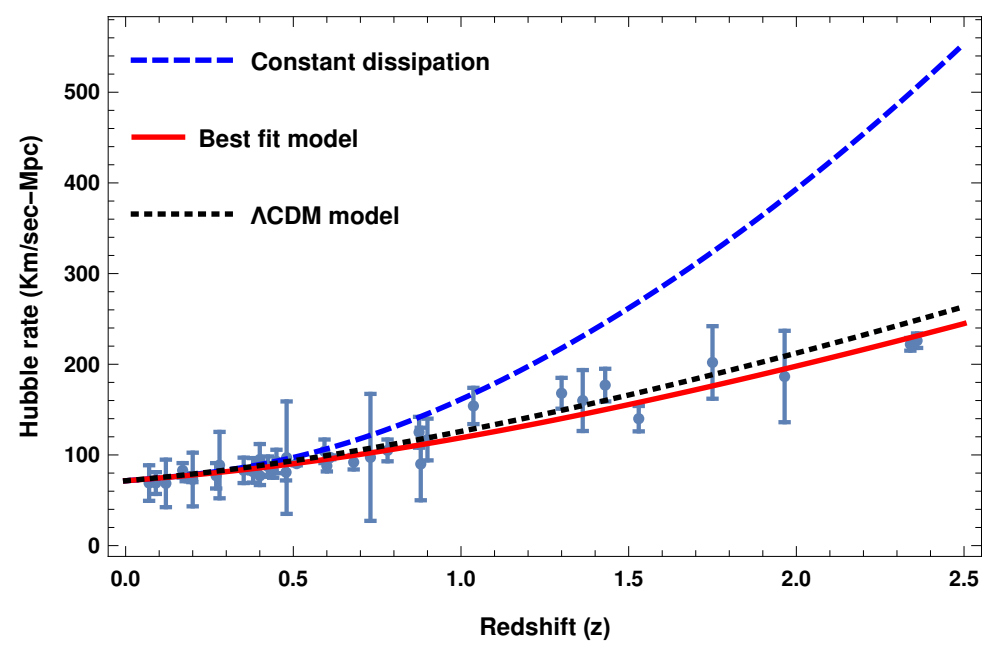

FIG. 2: Hubble parameter for the best fit value along with the case of constant dissipation is plotted. We also compare it with the $\Lambda$ CDM model.

fit and $\Lambda$ CDM model explain the Hubble data very well. Thus we find that the constant dissipation case doesn't explain the Hubble data.

\section{B. Fitting of Supernovae data}

Using the Hubble rate obtained in section IV, we calculate the luminosity distance, $d_{L}$, given as

$$
d_{L}(z)=\frac{(1+z)}{H_{0}} \int \frac{d z}{\bar{H}(z)} .
$$

The quantity measured in supernova observations is distance modulus $\mu$, which is related with the luminosity distance in the following manner,

$$
\mu(z) \equiv m-M=5 \log _{10}\left(\frac{\bar{d}_{L}(z)}{M p c}\right)+25,
$$

where $\bar{d}_{L}(z) \equiv H_{0} d_{L}(z)$ and $m, M$ represent apparent and absolute magnitude of type Ia supernovae.

In Fig. 3, we plot the distance modulus $(m-M)$ for the best fit value of model parameters $(n, L)$ and plot along with the Supernovae data taken from the Ref. [44, 45]. For a comparison, we also plot the distance modulus obtained for case of constant dissipation (for $n=0$ ). We see that the best fit and the constant dissipation case (where the velocity gradient is constant), match quite well on small redshift $(z \leq 1)$, but at large redshift $(z \geq 1)$, the difference between the two cases becomes prominent. We thus conclude that the decreasing 


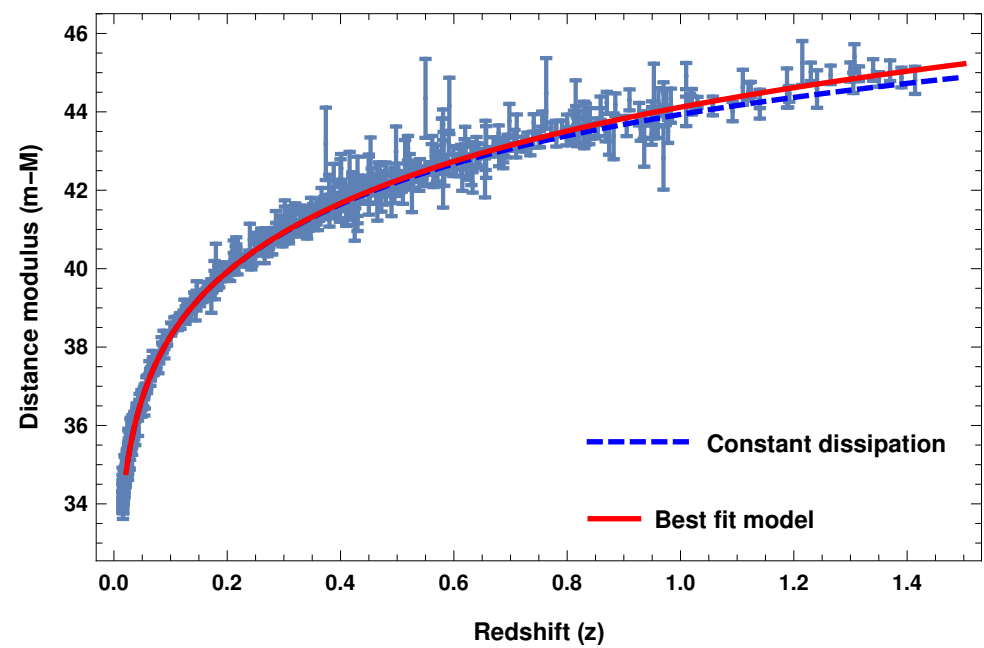

FIG. 3: Distance modulus $(m-M)$ obtained from best fit model parameters and constant dissipation have been plotted with the supernovae data. Our viscous SIDM explains the supernova data

dissipation with the redshift can explain both the cosmic chronometer and the supernova data while constant dissipations fails to do so.

\section{Deceleration parameter (q)}

To see the epoch of decelerated to accelerated phase transition (i.e. epoch of transition from $q>0$ to $q<0)$ of the Universe in viscous SIDM model, we plot $q(z)$ obtained from best fit value of the model parameters as shown in Fig. 4. To compare our results with the standard $\Lambda \mathrm{CDM}$ and constant dissipation scenario, we have also plotted the $q(z)$ obtained from the same.

We see that in viscous SIDM model, the transition point $z_{\operatorname{tr}} \sim 0.8$ and in $\Lambda$ CDM transition point $z_{\operatorname{tr}} \sim 0.7$. The transition point of constant dissipation case is $z_{\operatorname{tr}} \sim 0.3-0.4$ which is later in comparison with the $\Lambda$ CDM and viscous SIDM model. We also note that for constant dissipation, the deceleration parameter increases drastically and settles around $q \sim 1.2$ for higher $z$. This is quite in contrast with our expectation that $q$ should approach 0.5 in the matter dominated era. On the other hand, the value of deceleration parameter $q$ for best fit model parameter in viscous SIDM model saturates at $q \sim 0.4$ which is approximately same as the $\Lambda$ CDM prediction. We may thus safely conclude that the case for $n=0$, or constant dissipation, is surely not the case to appropriately describe the cosmic evolution. 


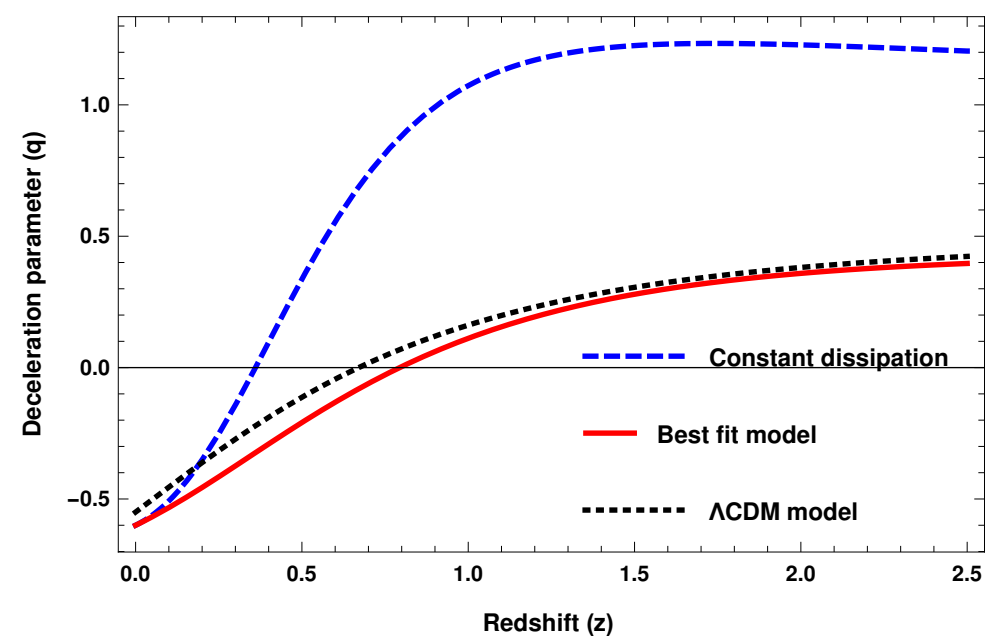

FIG. 4: Plot of deceleration parameter $(q)$ obtained from best fit along with the $q$ values for $\Lambda \mathrm{CDM}$ and constant dissipation case. The best fit model and $\Lambda \mathrm{CDM}$ appraoch $q=0.5$ in the matter dominated era.

\section{Dissipation parameter $(\beta)$}

As we have seen earlier in section III B that the dissipation parameter $\beta$, contains the information about the dissipative effect of viscous SIDM due to dark matter self interactions. To see the variation of dissipative effects, we plot the dissipation parameter $(\beta)$ as a function of redshift which is shown in Fig. 5 .

We see that for $n=0$ case, the velocity gradient and viscous coefficients are constant and hence the $\beta$ is constant. Thus the dissipative effects remain prominent even on the larger redshift. On the other hand, for the best fit value of model parameters that explain the data well, $\beta$ decreases sharply and becomes small for large $z$. This is not a surprising behaviour because in past when structures were being formed, the average peculiar velocity gradients were small, and consequently the dissipation term was not much effective. However in the later time of cosmic evolution, the density perturbations start growing which in turn increases the average peculiar velocity gradient and make the dissipation term important. Hence we find that the contribution of viscous effects becomes important at a late time not in early time.

From eq. (20) and (22) we note that at present epoch $\beta=\frac{4 \pi G D}{3 H_{0}^{3}}$, which is $\sim 4.1$ for our case. We see that our present estimated value, differs from the value $\left(\frac{4 \pi G D}{3 H_{0}^{3}} \sim 3.5\right)$ reported in Ref. [30]. Their conclusion was based on the assumption that at the present epoch 


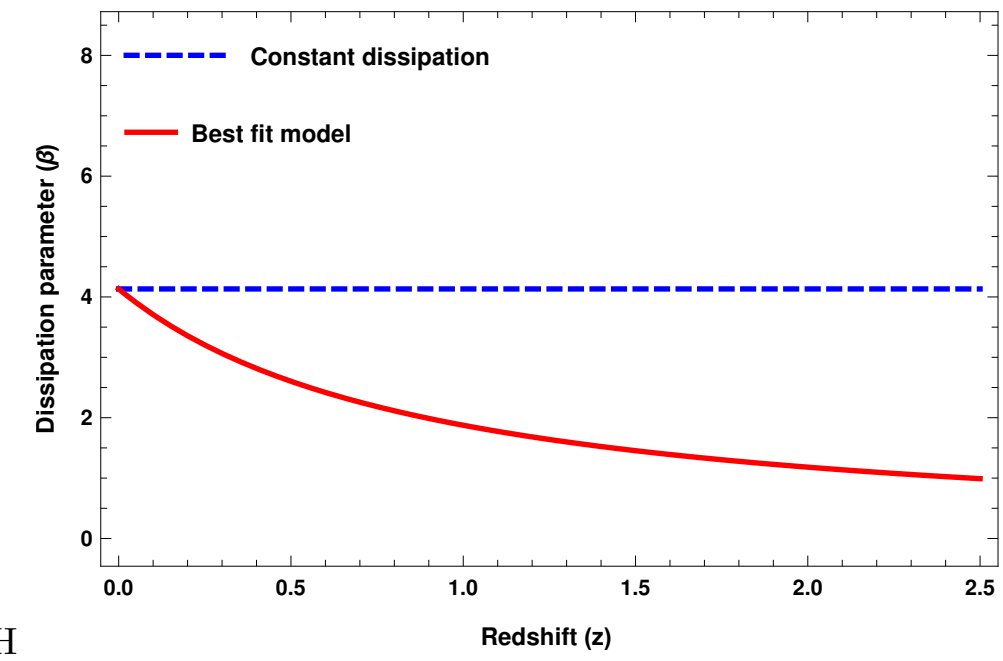

FIG. 5: Dissipation parameter $(\beta)$ obtained from best fit model parameters of our viscous SIDM model have been plotted along with the constant dissipation model. In farmer the dissipation is decreasing and in later the dissipation is constant with the redshift.

$\frac{d q}{d z}<<1$. In our work, by using the simplified assumptions as discussed in section III A, we find $\frac{d q}{d z}=0.61$. Hence by incorporating this contribution, we can explain the discrepancy between the $\beta$ values.

\section{SUMMARY AND CONCLUSION}

The viscous effect due to dark matter self-interactions may play an important role in the evolution history of the Universe. In late time the viscous effects of SIDM becomes important and may explain the present observed accelerated expansion of the Universe and hence mimic dark energy. In this work, we have tried to explain the cosmic evolution on the redshift range $0 \leq z \leq 2.5$ by incorporating the viscous effects in cosmic fluid which arise due to dark matter self interactions.

Assuming the cluster scale to be virialized and viscous coefficients are constant over the redshift of our interest $(0 \leq z \leq 2.5)$, we calculate the viscosity of SIDM using the Astrophysical constraints. Spatial-averages of velocity gradients are modelled to have a power law dependence on redshift. With these approximations we set up the equations governing the Hubble expansion rate and deceleration parameter. We estimate the best fit model parameters $(n, L)$ of our viscous SIDM model using $\chi^{2}$ minimization with the cosmic 
chronometer data.

We find the best fit values $n=0.5770$ and $L=20.1265$ explain the supernovae data and also attain the appropriate $q$ value in matter dominated era. We have also estimated the transition point of decelerated to accelerated expansion (i.e. epoch of $q>0$ to $q<0$ ) and found that the epoch of transition $z_{\mathrm{tr}} \sim 0.8$. The expectation that velocity gradients and hence dissipation should decrease at higher redshift and becomes important at late times in order to explain the present observed cosmic acceleration reflects in our viscous SIDM model for best fit parameters. Furthermore, we also compare the best fit model with the case of constant dissipation and find that the constant dissipation does not explain the data (cosmic chronometer and supernova) and also does not attain the correct value of deceleration parameter in matter dominated era. Our analysis is independent of any specific particle physics motivated model of SIDM.

\section{ACKNOWLEDGEMENT}

We would like to thank Subhendra Mohanty for useful discussions and comments. AM would also like to thank Richa Arya, Sampurn Anand and Jerin Mohan ND for fruitful discussions and suggestions. AA is financially supported by Scientific Education Research Board (SERB), under the National Post-Doctoral Fellowship (NPDF) grant number $\mathrm{PDF} / 2017 / 000641$.

[1] A. G. Riess et al. [Supernova Search Team], "Observational evidence from supernovae for an accelerating universe and a cosmological constant," Astron. J. 116, 1009 (1998) [astro$\mathrm{ph} / 9805201]$.

[2] S. Perlmutter et al. [Supernova Cosmology Project Collaboration], "Measurements of Omega and Lambda from 42 high redshift supernovae," Astrophys. J. 517, 565 (1999) [astro$\mathrm{ph} / 9812133]$.

[3] D. J. Eisenstein et al. [SDSS Collaboration], "Detection of the Baryon Acoustic Peak in the Large-Scale Correlation Function of SDSS Luminous Red Galaxies," Astrophys. J. 633, 560 (2005) [astro-ph/0501171]. 
[4] E. Komatsu et al. [WMAP Collaboration], "Seven-Year Wilkinson Microwave Anisotropy Probe (WMAP) Observations: Cosmological Interpretation," Astrophys. J. Suppl. 192, 18 (2011) [arXiv:1001.4538 [astro-ph.CO]].

[5] M. Tegmark et al. [SDSS Collaboration], "Cosmological Constraints from the SDSS Luminous Red Galaxies," Phys. Rev. D 74, 123507 (2006) [astro-ph/0608632].

[6] E. J. Copeland, M. Sami and S. Tsujikawa, "Dynamics of dark energy," Int. J. Mod. Phys. D 15, 1753 (2006) [hep-th/0603057].

[7] J. Yoo and Y. Watanabe, "Theoretical Models of Dark Energy," Int. J. Mod. Phys. D 21, 1230002 (2012) [arXiv:1212.4726 [astro-ph.CO]].

[8] S. Weinberg, "The Cosmological Constant Problem," Rev. Mod. Phys. 61, 1 (1989).

[9] T. Padmanabhan and S. M. Chitre, "Viscous universes," Phys. Lett. A 120, 433 (1987).

[10] O. Gron, "Viscous inflationary universe models," Astrophys. Space Sci. 173, 191 (1990).

[11] B. Cheng, "Bulk viscosity in the early universe," Phys. Lett. A 160, 329 (1991).

[12] W. Zimdahl, "Bulk viscous cosmology," Phys. Rev. D 53, 5483 (1996) [astro-ph/9601189].

[13] J. C. Fabris, S. V. B. Goncalves and R. de Sa Ribeiro, "Bulk viscosity driving the acceleration of the Universe," Gen. Rel. Grav. 38, 495 (2006) [astro-ph/0503362].

[14] A. Avelino and U. Nucamendi, "Can a matter-dominated model with constant bulk viscosity drive the accelerated expansion of the universe?," JCAP 0904, 006 (2009) [arXiv:0811.3253 [gr-qc]].

[15] N. D. J. Mohan, A. Sasidharan and T. K. Mathew, "Bulk viscous matter and recent acceleration of the universe based on causal viscous theory," Eur. Phys. J. C 77, no. 12, 849 (2017) [arXiv:1708.02437 [gr-qc]].

[16] N. Cruz, E. Gonzalez, S. Lepe and D. Saez-Chillon Gomez, "Analysing dissipative effects in the $\Lambda$ CDM model," arXiv:1807.10729 [gr-qc].

[17] S. Das and N. Banerjee, "Can neutrino viscosity drive the late time cosmic acceleration?," Int. J. Theor. Phys. 51, 2771 (2012) [arXiv:0806.3666 [gr-qc]].

[18] J. S. Gagnon and J. Lesgourgues, "Dark goo: Bulk viscosity as an alternative to dark energy," JCAP 1109, 026 (2011) [arXiv:1107.1503 [astro-ph.CO]].

[19] G. J. Mathews, N. Q. Lan and C. Kolda, "Late Decaying Dark Matter, Bulk Viscosity and the Cosmic Acceleration," Phys. Rev. D 78, 043525 (2008) [arXiv:0801.0853 [astro-ph]].

[20] B. Li and J. D. Barrow, "Does Bulk Viscosity Create a Viable Unified Dark Matter Model?," 
Phys. Rev. D 79, 103521 (2009) [arXiv:0902.3163 [gr-qc]].

[21] C. M. S. Barbosa, J. C. Fabris, O. F. Piattella, H. E. S. Velten and W. Zimdahl, "Viscous Cosmology," arXiv:1512.00921 [astro-ph.CO].

[22] O. F. Piattella, J. C. Fabris and W. Zimdahl, "Bulk viscous cosmology with causal transport theory," JCAP 1105, 029 (2011) [arXiv:1103.1328 [astro-ph.CO]].

[23] H. Velten and D. J. Schwarz, "Constraints on dissipative unified dark matter," JCAP 1109, 016 (2011) [arXiv:1107.1143 [astro-ph.CO]].

[24] S. Anand, P. Chaubal, A. Mazumdar and S. Mohanty, "Cosmic viscosity as a remedy for tension between PLANCK and LSS data," JCAP 1711, no. 11, 005 (2017) [arXiv:1708.07030 [astro-ph.CO]].

[25] S. Anand, P. Chaubal, A. Mazumdar, S. Mohanty and P. Parashari, JCAP 1805, no. 05, 031 (2018) doi:10.1088/1475-7516/2018/05/031 [arXiv:1712.01254 [astro-ph.CO]].

[26] G. Goswami, G. K. Chakravarty, S. Mohanty and A. R. Prasanna, "Constraints on cosmological viscosity and self interacting dark matter from gravitational wave observations," Phys. Rev. D 95, no. 10, 103509 (2017) [arXiv:1603.02635 [hep-ph]].

[27] R. G. Cai, T. B. Liu and S. J. Wang, "Gravitational wave as probe of superfluid dark matter," Phys. Rev. D 97, no. 2, 023027 (2018) [arXiv:1710.02425 [hep-ph]].

[28] B. Q. Lu, D. Huang, Y. L. Wu and Y. F. Zhou, "Damping of gravitational waves in a viscous Universe and its implication for dark matter self-interactions," arXiv:1803.11397 [astroph.HE].

[29] I. Brevik, . Grn, J. de Haro, S. D. Odintsov and E. N. Saridakis, "Viscous Cosmology for Earlyand Late-Time Universe," Int. J. Mod. Phys. D 26, no. 14, 1730024 (2017) [arXiv:1706.02543 $[\mathrm{gr}-\mathrm{qc}]]$.

[30] S. Floerchinger, N. Tetradis and U. A. Wiedemann, "Accelerating Cosmological Expansion from Shear and Bulk Viscosity,” Phys. Rev. Lett. 114, no. 9, 091301 (2015) [arXiv:1411.3280 $[\mathrm{gr}-\mathrm{qc}]]$.

[31] A. Atreya, J. R. Bhatt and A. Mishra, "Viscous Self Interacting Dark Matter and Cosmic Acceleration," JCAP 1802, no. 02, 024 (2018) [arXiv:1709.02163 [astro-ph.CO]].

[32] D. N. Spergel and P. J. Steinhardt, "Observational evidence for selfinteracting cold dark matter," Phys. Rev. Lett. 84, 3760 (2000) [astro-ph/9909386].

[33] S. Tulin and H. B. Yu, "Dark Matter Self-interactions and Small Scale Structure," Phys. Rept. 
730, 1 (2018) [arXiv:1705.02358 [hep-ph]].

[34] M. Kaplinghat, S. Tulin and H. B. Yu, "Dark Matter Halos as Particle Colliders: Unified Solution to Small-Scale Structure Puzzles from Dwarfs to Clusters," Phys. Rev. Lett. 116, no. 4, 041302 (2016) [arXiv:1508.03339 [astro-ph.CO]].

[35] S. Gavin, "Transport Coefficients In Ultrarelativistic Heavy Ion Collisions," Nucl. Phys. A 435, 826 (1985).

[36] G. P. Kadam and H. Mishra, "Dissipative properties of hot and dense hadronic matter in an excluded-volume hadron resonance gas model," Phys. Rev. C 92, no. 3, 035203 (2015)

[37] M. Weber and W. de Boer, "Determination of the Local Dark Matter Density in our Galaxy," Astron. Astrophys. 509, A25 (2010) [arXiv:0910.4272 [astro-ph.CO]].

[38] R. Kuzio de Naray, S. S. McGaugh and W. J. G. de Blok, "Mass Models for Low Surface Brightness Galaxies with High Resolution Optical Velocity Fields," Astrophys. J. 676, 920 (2008) [arXiv:0712.0860 [astro-ph]].

[39] A. B. Newman, T. Treu, R. S. Ellis, D. J. Sand, C. Nipoti, J. Richard and E. Jullo, "The Density Profiles of Massive, Relaxed Galaxy Clusters: I. The Total Density Over 3 Decades in Radius," Astrophys. J. 765, 24 (2013) [arXiv:1209.1391 [astro-ph.CO]].

[40] A. B. Newman, T. Treu, R. S. Ellis and D. J. Sand, "The Density Profiles of Massive, Relaxed Galaxy Clusters: II. Separating Luminous and Dark Matter in Cluster Cores," Astrophys. J. 765, 25 (2013) [arXiv:1209.1392 [astro-ph.CO]].

[41] A. K. Mishra, Manuscript under preperation.

[42] P. A. R. Ade et al. [Planck Collaboration], "Planck 2013 results. XVI. Cosmological parameters," Astron. Astrophys. 571, A16 (2014) [arXiv:1303.5076 [astro-ph.CO]].

[43] O. Farooq, F. R. Madiyar, S. Crandall and B. Ratra, "Hubble Parameter Measurement Constraints on the Redshift of the Decelerationacceleration Transition, Dynamical Dark Energy, and Space Curvature," Astrophys. J. 835, no. 1, 26 (2017) [arXiv:1607.03537 [astro-ph.CO]].

[44] R. Amanullah et al., "Spectra and Light Curves of Six Type Ia Supernovae at 0.511 ; z ; 1.12 and the Union2 Compilation," Astrophys. J. 716, 712 (2010) [arXiv:1004.1711 [astro-ph.CO]].

[45] N. Suzuki et al. [Supernova Cosmology Project Collaboration], "The Hubble Space Telescope Cluster Supernova Survey: V. Improving the Dark Energy Constraints Above z¿1 and Building an Early-Type-Hosted Supernova Sample," Astrophys. J. 746, 85 (2012) [arXiv:1105.3470 [astro-ph.CO]]. 